\title{
Neonatal resuscitation and extracorporeal cardiopulmonary resuscitation: a dire need for developing countries
}

Keywords: neonatal resuscitation program (NRP), american academy of pediatrics (AAP), capacity building, ECPR

\section{Brief correspondence}

Reports suggested that neonatal deaths account for $40 \%$ of deaths under the age of 5years worldwide. Two-thirds of the world's neonatal deaths occur in just 10countries, mostly in Asia where country like Pakistan stands the third highest neonatal mortality rate in the world. ${ }^{1}$ In Pakistan, prematurity and birth asphyxia are the leading causes of neonatal death and are closely linked to obstetric factors. ${ }^{1,2}$ Neonatal resuscitation (NRP) of the American Academy of Pediatrics, conducted at the time of birth to help the newborn in setting up breathing and circulation, thus plays a pivotal role. ${ }^{3}$ The basic steps in resuscitation process are critical for reducing neonatal morbidity and mortality. Quite often the basic NRP remains inadequate to contribute for standard care and hence neonatal deaths remains inevitable. Previously, reports have suggested that extracorporeal membrane oxygenation (ECMO) support instituted as rescue therapy during CPR (E-CPR) in children who had cardiopulmonary arrest and failed to respond to conventional NRP promoted survival..$^{3-5}$ ECPR, allows clinicians to potentially rescue patients unresponsive to traditional CPR. Since then, E-CPR has been increasingly used to rescue children with both primary cardiac and no cardiac diseases receiving CPR after conventional resuscitative measures have failed to restore adequate circulation. Extracorporeal life support organization (ELSO) reported in 2004, 151 neonatal cases and 282 pediatric cases of ECPR. ${ }^{6}$ Among these cases, the neonatal ECPR survival rate to discharge was $43 \%$, and the pediatric ECPR survival rate to discharge was $39 \% .{ }^{6}$ Though favorable survival outcomes for E-CPR have been demonstrated in many reports, ECMO is neither universally available nor accepted into clinical practice, and the current reports on the effectiveness of E-CPR are institution-specific. Realizing this, we, at the HeartLung \& Research Institute, Cardiac Eye International Foundation (CEIF) has for the first time commenced the Neonatal and Pediatric Extracorporeal Membrane Oxygenation (ECMO) Program. ECMO is partial prolonged heart lung bypass for selected infants and children with life-threatening respiratory failure due to severe, yet reversible cardiac or pulmonary disease. The addition of our ECMO program to other existing advanced technology therapies including advanced NRP has allowed to provide the full spectrum of advanced cardiopulmonary support services for infants and children in Pakistan.

The most likely E-CPR candidates are term or nearterm infants with respiratory failure secondary to:
i. Meconium aspiration syndrome
ii. Persistent pulmonary hypertension
iii. Neonatal sepsis/pneumonia

Hence patient populations benefiting from E-CPR are:

i. Respiratory distress syndrome
Volume 4 Issue I - 2017

\author{
Maqsood M Elahi \\ Heart-Lung \& Research Institute, Cardiac Eye International \\ Foundation, USA \\ Correspondence: Maqsood Elahi, Distinguished Professor \\ of Cardiovascular Sciences, Adult \& Pediatric Cardiothoracic \\ Surgeon, Founder \& Chairman Cardiac Eye International \\ Foundation, Texas, USA, Tel + 254- 458- 765-I, \\ Email manzoor_elahi@hotmail.com
}

Received: January 07, 2017 | Published: January 25, 2017

ii. Congenital diaphragmatic hernia

iii. Severe cardiac or cardiopulmonary failure

iv. Gestational age $>34$ weeks

v. Birth weight $>2,000$ grams

vi. Reversible lung disease (mechanical ventilation $<10-14$ days)

vii. No major intracranial hemorrhage (Grade 1 or less)

viii. No uncorrectable congenital cardiac lesion

ix. No lethal congenital anomalies

x. No significant coagulopathy or uncontrolled bleeding complications

xi. Pneumonia

xii. Bronchiolitis

xiii. Sepsis/septic shock

xiv. Adult respiratory distress syndrome (ARDS)

$\mathrm{xv}$. Aspiration syndromes

xvi. Intrapulmonary hemorrhage

xvii. Reversible lung disease (mechanical ventilation $<7-10$ days)

xviii. No significant coagulopathy or uncontrolled bleeding complications

xix. No permanent, severe central nervous system injury.

The CEIF E-CPR Program is directed by ECMO-trained neonatologists and pediatric intensivists and supported by the cardiothoracic surgeon. We have developed a highly professional multi-disciplinary team to care for E-CPR patients around the clock. Pediatric cardiologists and neurologists are available for initial consultations and subsequent follow up. A team of highly-trained ECMO specialists provides circuits management under the direction 
and supervision of the surgeon. An ECMO follow-up program has been developed at Heart- Lung \& Research Institute to evaluate the neurologic and neuro developmental progress of these patients. ECMO patient selection and their subsequent referral and transport are closely associated without come. In most cases, deteriorating oxygenation will respond to increasing ventilatory support, but the progression towards respiratory failure in these critically-ill patients is far from linear and may further progress to refractory hypoxic cardiac arrest.

In summary, to address the challenges in reducing the rate of childhood mortality, there is a need for better understanding of the neonatal resuscitation skills. Further to it, to decrease the risk of death, E-CPR should be considered as a treatment modality prior to when criteria are met. Although making the decision to transfer a patient for E-CPR may be difficult, early consolation with the ECMO physician at CEIF can greatly facilitate this process. We believe that maintenance and improvement of neonatal care require active involvement of all the staff working in healthcare system. They should also be considered essential members of the healthcare system, providing maternal and child health services at basic and rural health care units in Pakistan. Their training and capacity-building is of outmost importance to reduce the maternal and newborn morbidity and mortality.

\section{Conclusion}

There is a need for regular such in-service modalities regarding basic to advanced neonatal resuscitation in Pakistan; and it should be made a pre-requisite to get a job for the medical staff. Training should emphasize more on the practical component. Evidence-based neonatal resuscitation guidelines should be available at all healthcare levels. Neonatal resuscitation provided to any newborn should be properly documented along with its outcome. Newton and English reviewed the evidence for neonatal resuscitation and concluded that effective resuscitation was possible with basic equipment and skills in low-resource settings. Therefore, universal application of resuscitation including E-CPR may save hundreds of thousands of newborn lives currently lost each year, and contribute significantly to progress toward Millennium Development Goal $4 .^{8}$ To achieve impact, the challenge is to improve neonatal resuscitation where resources are limited and where many, even most, babies are born at home. The curriculum courses such as devised by AAP should play a significant portion about neonatal resuscitation and must be given due importance in Pakistan's health care system. Neonatal resuscitation training including E-CPR should be incorporated into national neonatal strategies to complement the top priority of improved prevention of intrapartum-related deaths through obstetric care. ${ }^{9}$

\section{Acknowledgements}

None.

\section{Conflict of interest}

The author declares no conflict of interest.

\section{References}

1. Alkema L, Jin Rou New, Pedersen J, et al. Levels and trends in child mortality, 2015 Report, Estimates developed by UN Interagency group for child mortality estimation. PLoS One. 2014;9(7).

2. Imtiaz J, Hillary H, Sohail S, et al. Neonatal mortality, risk factors and causes: a prospective population-based cohort study in urban Pakistan. Bull World Health Organ. 2009;87(2):130-138.

3. Alsouf B, Al-Radi OO, Nazer RI, et al. Survival outcomes after rescue extracorporeal cardiopulmonary resuscitation in pediatric patients with refractory cardiac arrest. J Thorac Cardiovasc Surg. 2007;134(4):952-959.

4. Morris MC, Wernovsky G, Nadkarni VM. Survival outcomes after extracorporeal cardiopulmonary resuscitation instituted during active chest compressions following refractory in-hospital pediatric cardiac arrest. Pediatr Crit Care Med. 2004;5(5):440-446.

5. Thiagarajan RR, Laussen PC, Rycus PT, et al. SL Extracorporeal membrane oxygenation to aid cardiopulmonary resuscitation in infants and children. Circulation. 2007;116(15):1693-1700.

6. Conrad SA, Rycus PT, Dalton H. Extracorporeal life support registry report 2004. ASAIO J. 2005;51(1):4-10.

7. Newton O, English M. Newborn resuscitation: defining best practice for low-income settings. Trans R Soc Trop Med Hyg. 2006;100(10):899-908.

8. Vali P, Mathew B, Lakshminrusimha S. Neonatal resuscitation: evolving strategies. Matern Health Neonatol Perinato. 2015;1:4.

9. Hofmeyr J, Haws RA, Bergstrom S, et al. Obstetric care in low-resource settings: what, who, how, and overcoming challenges to scale up. Int $J$ Gynecol Obstet. 2009;107(1):21-45. 